\title{
Compounding a High Dielectric Constant Thermoplastic Material for Production of Microwave Photonic Crystals through Additive Manufacturing
}

\author{
Gerardo A. Mazzei Capote ${ }^{1}$, Maria Camila Montoya-Ospina ${ }^{1}$, Zijie Liu루, Michael S. Mattei², \\ Boyuan Liu ${ }^{3}$, Aidan P. Delgado ${ }^{2}$, Zongfu Yu ${ }^{3}$, Randall H. Goldsmith², Tim A. Osswald ${ }^{1}$
}

1. Department of Mechanical Engineering, University of Wisconsin- Madison, Madison, WI 53706

2. Department of Chemistry, University of Wisconsin- Madison, Madison, WI 53706

3. Department of Electrical and Computer Engineering, University of Wisconsin-Madison, Madison, WI 53706

\begin{abstract}
:
Additive Manufacturing techniques allow the production of complex geometries unattainable through other traditional technologies. This advantage lends itself well to rapidly iterate and improve upon the design of microwave photonic devices, which are structures with intricate, repeating features. The issue tackled by this work involves compounding a high-dielectric constant material that can be used to produce 3D topological structures using polymer extrusionbased AM techniques. This material was ABS based, and used barium titanate ceramic as the high-dielectric compound of the composite, and involved the use of a surfactant and a plasticizer to facilitate processing. Initial small amounts of material were compounded using an internal batch mixer, and studied using polymer thermal analysis techniques, such as thermogravimetric analysis, rheometry, and differential scanning calorimetry to determine the proper processing conditions. The production of the material was then scaled-up through the use of a twin-screw extruder system, producing homogeneous pellets. Finally, the thermoplastic composite was used with a screw-based, material extrusion additive manufacturing technique to produce a slab for measuring the dielectric constant of the material, as well as a preliminary 3D photonic crystal. The real part of dielectric constant of the composite was measured to be 12.85 in the range of $10 \mathrm{GHz}$ to $12 \mathrm{GHz}$, representing the highest dielectric constant ever demonstrated for a thermoplastic AM composite at microwave frequencies. The dielectric loss tangent was equal to 0.046 , representing a low-loss dielectric.
\end{abstract}

\section{Introduction:}

Additive Manufacturing (AM) techniques have risen to the forefront of engineering given their capacity of reproducing intricate design features that would otherwise be difficult to attain through traditional manufacturing processes. Additionally, the compressed part production cycle that comes attached to all AM technologies allows rapid iteration and optimization of part design. These advantages over traditional manufacturing are already being leveraged by certain industrial sectors, such as aerospace, to reduce the number of parts required for an assembly, or increasing the efficiency of processes by exploiting the capability of AM processes to reproduce complex geometric features. However, these advantages also lend themselves well to applications in the 
fields of photonics and telecommunication systems, particularly in applications where optimization of geometry is paramount to the success of the finished part. In this realm, production of microwave photonic devices represents an ideal challenge that can be solved through the use of AM.

Application of AM to microwave photonics has been leveraged to produce simple device designs such as microwave lenses and waveguides, as well as complex geometries of microwave photonic crystals and metamaterials [1]-[7] For these more complex device geometries, and especially for 3D photonic crystals (PCs), the need for high-dielectric constant materials to realize the desired photonic properties is an outstanding challenge [3], [8], [9]. Solution involving AM exist, requiring the use of customized material solutions to deliver the dielectric properties necessary for proper PC performance. Examples include the use of a ceramic suspension in photosensitive resin with the Stereolithography process [10]-[12], and the production of composite thermoplastic filaments to be used in Material Extrusion processes [13].

This research paper explores the compounding of a high-dielectric, thermoplastic material through twin-screw extrusion, and the subsequent manufacture of a preliminary PC structure using a hybrid AM machine that can extrude material in pellet form, as well as support material supplied as a thermoplastic filament. The composition of this material is based on a recipe published by $\mathrm{Wu}$, Isakov, and Grant [13], and similar findings describing the effect of $\mathrm{BaTiO}_{3}$ filler on dielectric properties of composite films [14] This work represents a case-study for implementation of AM techniques in the manufacture of PC structures, or other engineering applications that require a high dielectric material and reproduction of complex geometric structures.

\section{Materials and Methods:}

Raw Materials:

ABS CYCOLAC TM MG94 - SABIC (MFI: $11.7 \mathrm{~g} / 10 \mathrm{~min}, 230 \mathrm{C} / 3.8 \mathrm{Kg}$ )

BaTiO3 - 99\% purity, $<3$ micron powder - 35\% by volume - Sigma-Aldrich

Dibutyl Phthalate as plasticizer - $5 \%$ by volume - Sigma-Aldrich

Octyl Gallate as surfactant - $1 \%$ by weight - Sigma-Aldrich

\section{Preliminary testing:}

Mixing trials using a Brabender internal batch mixer:

The ABS/BaTiO3 composite was prepared in small batches using a C.W. Brabender 3-Piece mixing bowl attached to an Intelli Plasti-Corder Torque Rheometer, as a preliminary step to characterize the requirements of the compounding process. Mixing occurred using roller blades with a chamber volume of $60 \mathrm{~cm}^{3}$ and a fill factor of 0.7 . Results from this mixing experiment can be seen in Figure 1.

Two batches were prepared to study the effect of the plasticizer in the rheological properties of the composite. One batch contained plasticizer, and the other did not. The mixing was done in a 
multi-step protocol that proceeded as follows: (1) ABS pellets were added to the mixer, (2) after about 4.5 minutes, the BaTiO3 and octyl gallate $(\mathrm{OG})$ powder were added to the melt and mixed for an additional 3.5 minutes and (3) the plasticizer, dibutyl phthalate (DP), was added and mixed for 2 minutes. The addition of plasticizer was omitted in the second batch. All mixing steps were done at $190{ }^{\circ} \mathrm{C}$ and 50 RPM and the torque was monitored. After mixing, the material was compressed into $25 \mathrm{~mm}$ discs using a CARVER Auto Series NE press under 0.5 tons at $190{ }^{\circ} \mathrm{C}$ for 1 minute. These discs were used in the parallel plate rheometer to characterize thermal and rheological behavior of the material.

The effects that the ceramic filler $\left(\mathrm{BaTiO}_{3}\right)$ and the plasticizer have on the viscosity of the blend was determined by the torque curve shown in Figure 1. The torque, which is related to the viscosity, increases approximately by a factor of four when the ceramic is added to the ABS melt. The torque recorded at the 5 -minute mark was $22 \mathrm{~N} \cdot \mathrm{m}$. As the ceramic is dispersed in the polymer matrix, the torque decreases, approaching a plateau value of $14 \mathrm{~N} \cdot \mathrm{m}$, at which point the plasticizer is fed. The torque decreased from $14 \mathrm{~N} \cdot \mathrm{m}$ to $8 \mathrm{~N} \cdot \mathrm{m}$ in this step. This information provides a valuable guide for the torque requirements for the next two processing steps: compounding in a twin screw, and extrusion-based 3D printing.

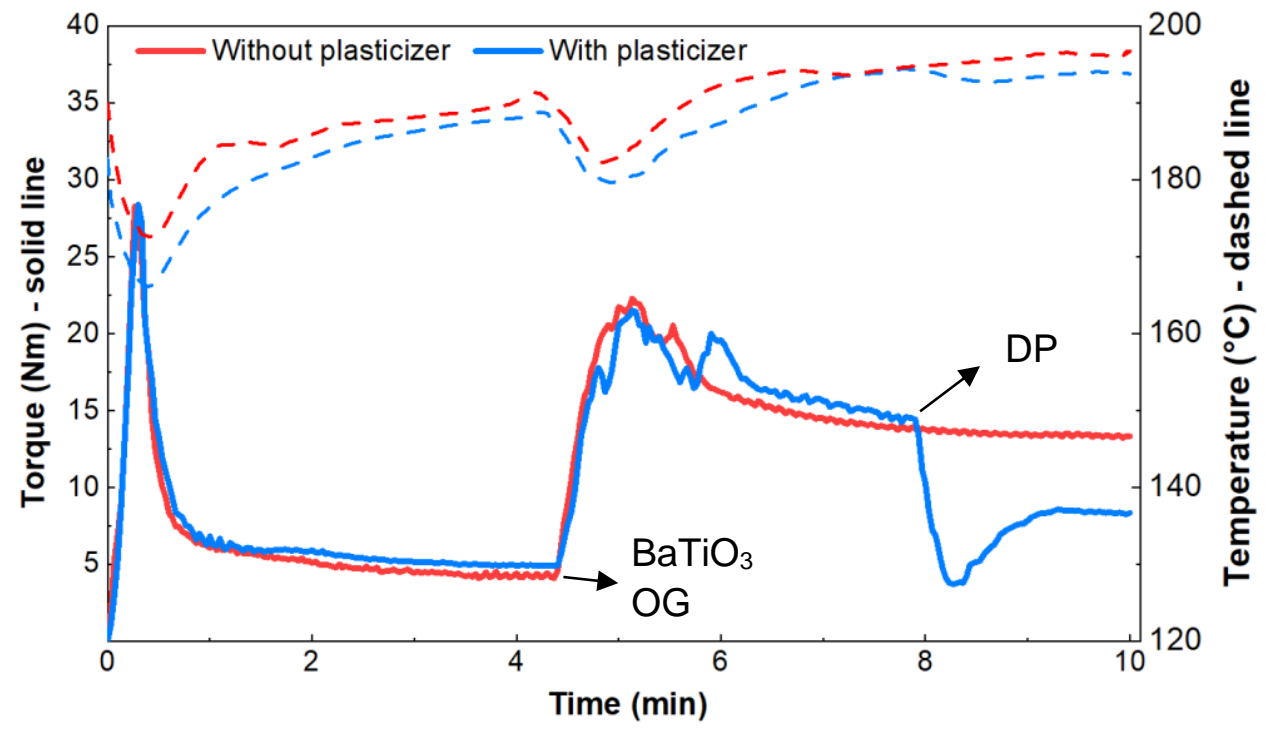

Figure 1: Internal mixing results comparing torque requirements of composite with and without plasticizer where the solid line is torque and dashed line is temperature $\left(50 \mathrm{rpm}, 190^{\circ} \mathrm{C}\right)$.

Thermogravimetric analysis (TGA) and Differential scanning calorimetry (DSC):

To understand the thermal stability and proper processing conditions of the ABS/BaTiO3 composite, thermogravimetric and calorimetric tests were conducted on the material. Thermogravimetric analysis was conducted using a NETZSCH TG 209 F1 Libra. Dynamic experiments were performed in an oxygen atmosphere. Samples were ramped from $25^{\circ} \mathrm{C}$ to $600.0^{\circ} \mathrm{C}$ at a heating rate of $10 \mathrm{~K} / \mathrm{min}$. 
Figure $2 \mathrm{a}$ shows the TGA results from the composites prepared in the internal mixer shown in Figure 1. The composite without plasticizer has a degradation onset temperature of $401.8^{\circ} \mathrm{C}$ which is attributed to the ABS matrix. The composite with plasticizer shows two degradation mechanisms. The first degradation onset temperature occurs at $176.0^{\circ} \mathrm{C}$ and the second one at $389.8^{\circ} \mathrm{C}$. The first mass loss is most likely due to the evaporation of the plasticizer. For this reason, the temperature profile selected in the twin screw extruder and in the 3D printer were set to values below $176.0^{\circ} \mathrm{C}$. The first derivative of the mass change with respect to temperature is shown in Figure $2 \mathrm{~b}$. Lastly, the residual mass measured at $600.0{ }^{\circ} \mathrm{C}$ was $75 \%$, corresponding to degradation of the ceramic.
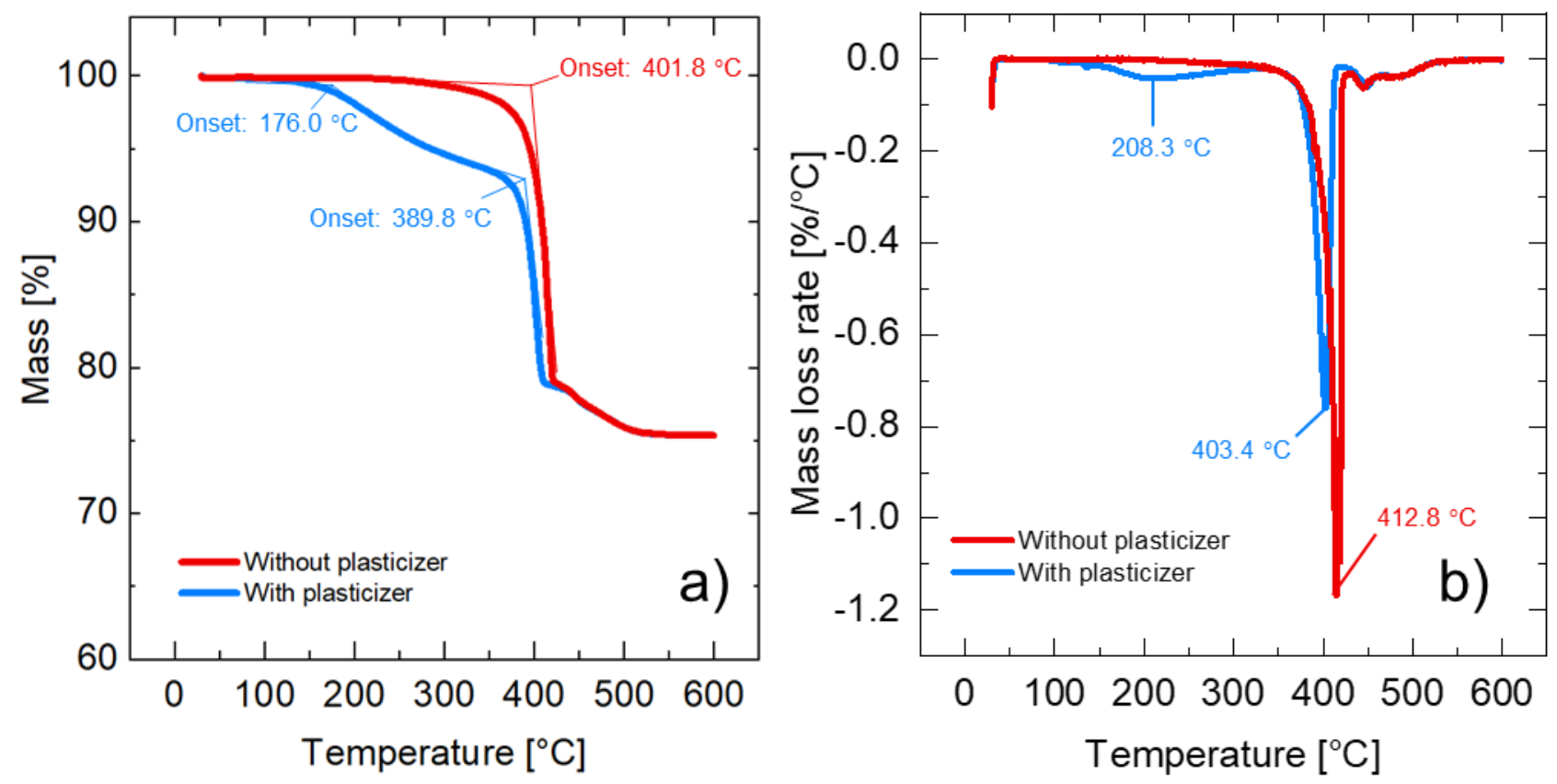

Figure 2: TGA measurements of ABS composite with and without plasticizer (10 K/min, Oxygen). a) Mass loss [\%] vs temperatures $\left[{ }^{\circ} \mathrm{C}\right]$ b) mass loss rate $\left[\% /{ }^{\circ} \mathrm{C}\right]$ vs temperature $\left[{ }^{\circ} \mathrm{C}\right]$.

Calorimetry experiments were performed with a NETZSCH DSC 214 Polyma. The samples were ramped from $20{ }^{\circ} \mathrm{C}$ to $150{ }^{\circ} \mathrm{C}$ at a heating rate of $10 \mathrm{~K} / \mathrm{min}$ under nitrogen. These tests were performed to establish the effect of the plasticizer and surfactant upon the glass transition temperature of the material. The glass transition temperature $\left(T_{g}\right)$ measured in the neat $A B S$ and in the composites with and without plasticizer is shown in Figure 3. The neat ABS has an onset $\mathrm{T}_{\mathrm{g}}$ of $103^{\circ} \mathrm{C}$ with a midpoint of $106^{\circ} \mathrm{C}$ corresponding to the styrene component. Moreover, the glass transition of ABS shows a clear relaxation peak. Enthalpy relaxation is a response of amorphous materials annealed below its glass transition temperature as previously demonstrated by X. Quan et al. [15], [16]. The addition of plasticizer had a significant effect on the glass transition temperature of the composite. The $\mathrm{T}_{\mathrm{g}}$ of the composite with plasticizer was not detected in the temperature range tested $\left(25^{\circ} \mathrm{C}-150^{\circ} \mathrm{C}\right)$. Due to limitations in the equipment, the temperature could not be further decreased. Notice that the addition of the surfactant (octyl gallate) also decreased the $T_{g}$ of the composite by approximately $10^{\circ} \mathrm{C}$. This result is aligned with the data reported by Wu et al. [13]. A melting point at $130.0^{\circ} \mathrm{C}, 131.5^{\circ} \mathrm{C}$ and $132.0^{\circ} \mathrm{C}$ was observed in the 
neat $\mathrm{ABS}$ and in the ABS composite without and with plasticizer, respectively. This value is consistent with the peak temperature of the acrylonitrile component. The rheological characterization in the parallel plate rheometer was performed at $160.0^{\circ} \mathrm{C}$. This temperature was selected after analyzing the TGA and DSC results.

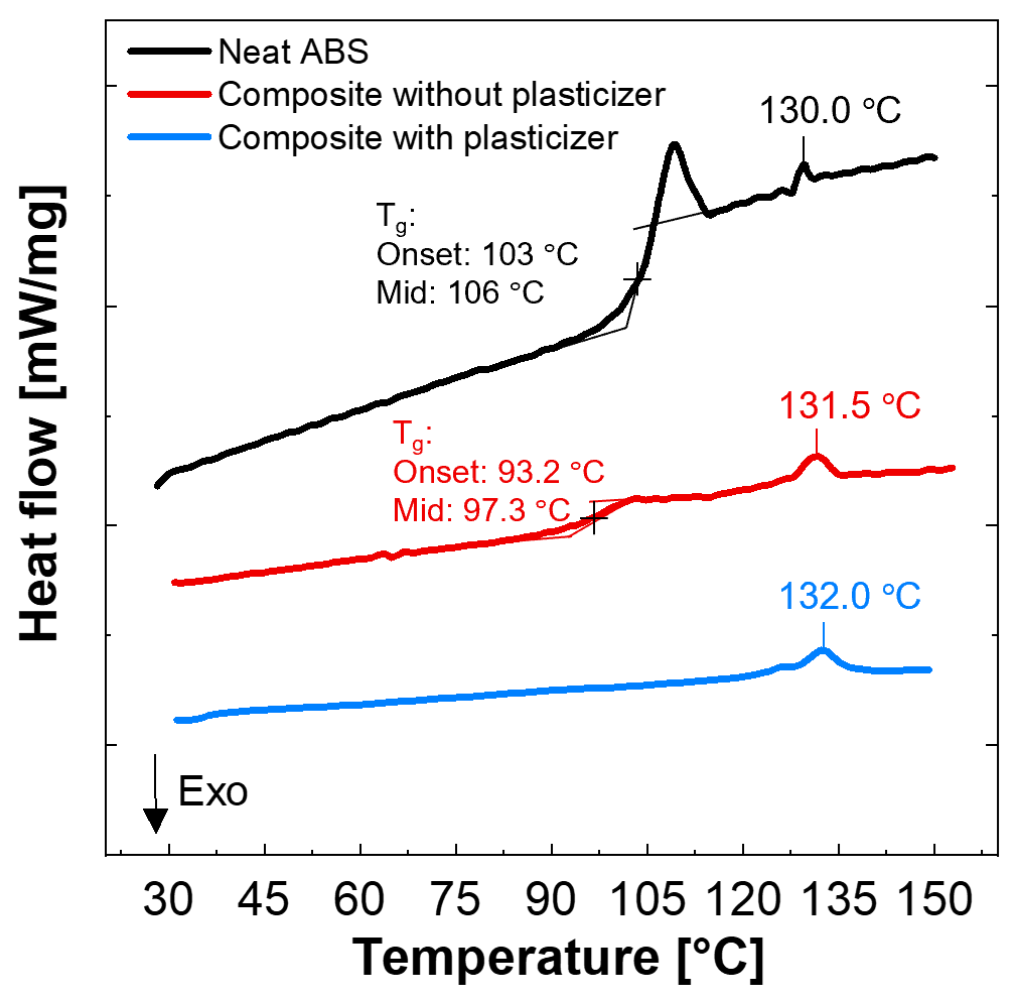

Figure 3: DSC heating curves of neat $A B S$ and $A B S$ composite without and with plasticizer $(10 \mathrm{~K} / \mathrm{min}$, nitrogen).

Parallel plate rheometer:

Rheology measurements were performed to investigate the properties of $A B S$ and the $A B S$ composites in the melt. These measurements were made on a TA Instruments AR 2000ex rheometer. A $25 \mathrm{~mm}$ parallel steel plate fixture was used to test the materials with a gap of 1.8 $\mathrm{mm}$. Strain sweeps were performed at a constant frequency of $1 \mathrm{rad} / \mathrm{s}$ and strains from $0.01 \%$ to $100 \%$. The strain sweeps were used to determine the linear viscoelastic region to be explored during small-amplitude oscillatory shear (SAOS) measurements. Frequency sweeps were then performed at $160^{\circ} \mathrm{C}$ at a strain of $0.1 \%$ and a frequency range of $0.1-100 \mathrm{rad} / \mathrm{s}$ and the results are shown in Figure 4.

At $100 \mathrm{rad} / \mathrm{s}$, the measured viscosities $\left(\left|\eta^{*}\right|\right)$ for ABS, ABS composite without plasticizer, and ABS composite with plasticizer were $3734 \mathrm{rad} / \mathrm{s}, 12750 \mathrm{rad} / \mathrm{s}$ and $1953 \mathrm{rad} / \mathrm{s}$, respectively. The high viscosity values recorded in the composite blend without plasticizer is due to the high content of ceramic filler (35\% wt.). However, by adding a plasticizer in the composite, the viscosity was 
decreased by a factor of 6.5 . In the frequency range of $0.5-100 \mathrm{rad} / \mathrm{s}$, the viscosity of the ABS composite with plasticizer was even lower than the neat ABS. For this reason, the former blend was pursued for the compounding step and 3D printing.

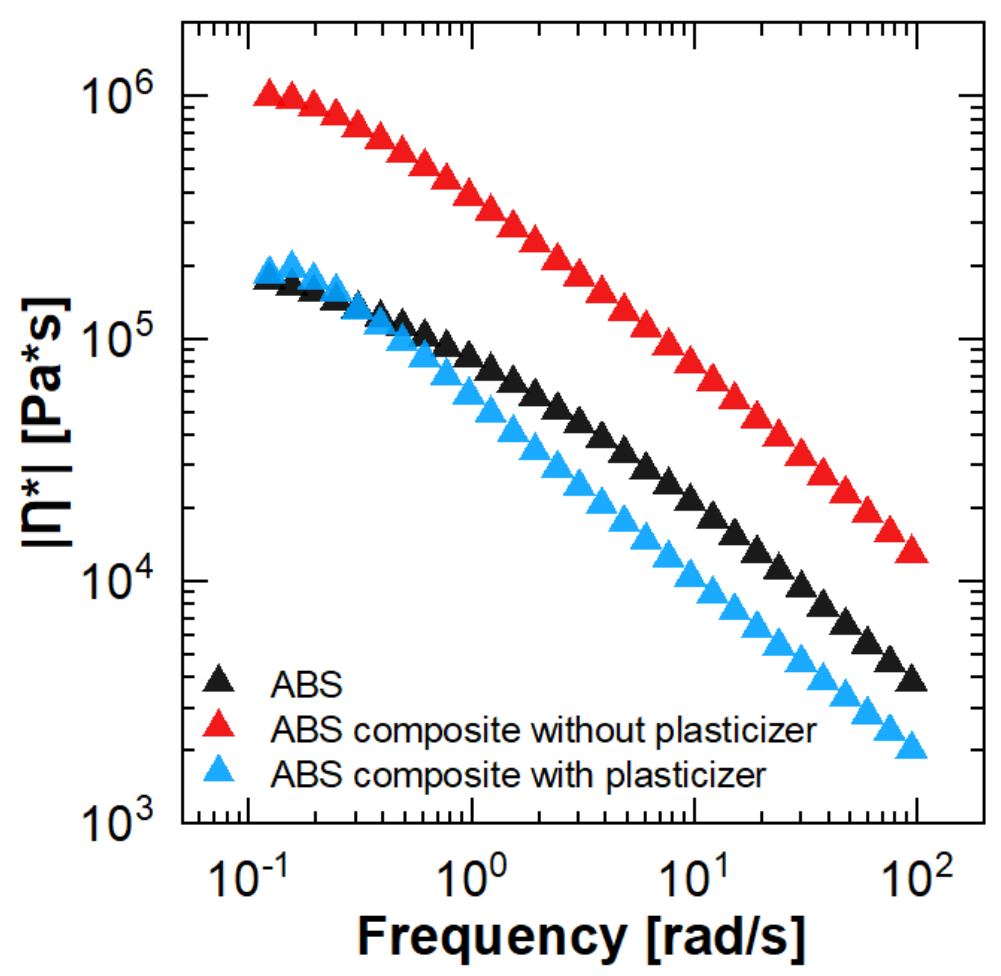

Figure 4: Complex viscosity $\left(\left|\eta^{*}\right|\right)$ of $A B S$ and $A B S$ composite without and with plasticizer $(0.1 \%$ strain, $\left.160^{\circ} \mathrm{C}\right)$.

\section{Material compounding:}

Twin screw extrusion

After the raw materials (ABS, BaTiO3, plasticizer, and surfactant) were pre-processed in an acetone solution, dried, and ground into a coarse powder using a blender, a twin screw extruder setup (Fig.5) was used to produce the feedstock for AM in the form of ABS composite pellets. The equipment consisted of a twin screw extruder (Leistritz ZSE 27) with $27 \mathrm{~mm}$ screw diameter, a L/D ratio of $36 \mathrm{D}$, and a die with a circular diameter of $3 \mathrm{~mm}$, followed by a water bath destined to cool the extrudate, and a pelletizer. A screw feeder (Schenck process Mechatron Coin-Flex Screw Feeder) allowed precise control of the material supply to the throat of the twin screw extruder. This setup was selected due to the enhanced mixing capabilities of the twin screw system over a traditional single screw setup, rooted in higher overall shear forces that allow deagglomerating the particles of the filler [17]. 
An optimal temperature profile for the composite was found using the results from the preliminary testing, and is listed in Table 1 . Note how the temperature is kept below $176^{\circ} \mathrm{C}$ to prevent volatilization of the components, as shown in the TGA results. The extruder speed and the feeding rate were selected to maintain the melt pressure under 500 psi. Extruder speed was set at 50 RPMs, and the material was supplied using a feed rate of $2 \mathrm{~kg} / \mathrm{hr}$.

Table 1: List of temperature profile

\begin{tabular}{|c|c|c|c|c|}
\hline Zone Section & Zones 1 \& 2 & Zone 3 & Zones 4 to 8 & Die \\
\hline Temperature $\left[{ }^{\circ} \mathrm{C}\right]$ & 120 & 125 & 130 & 130 \\
\hline
\end{tabular}

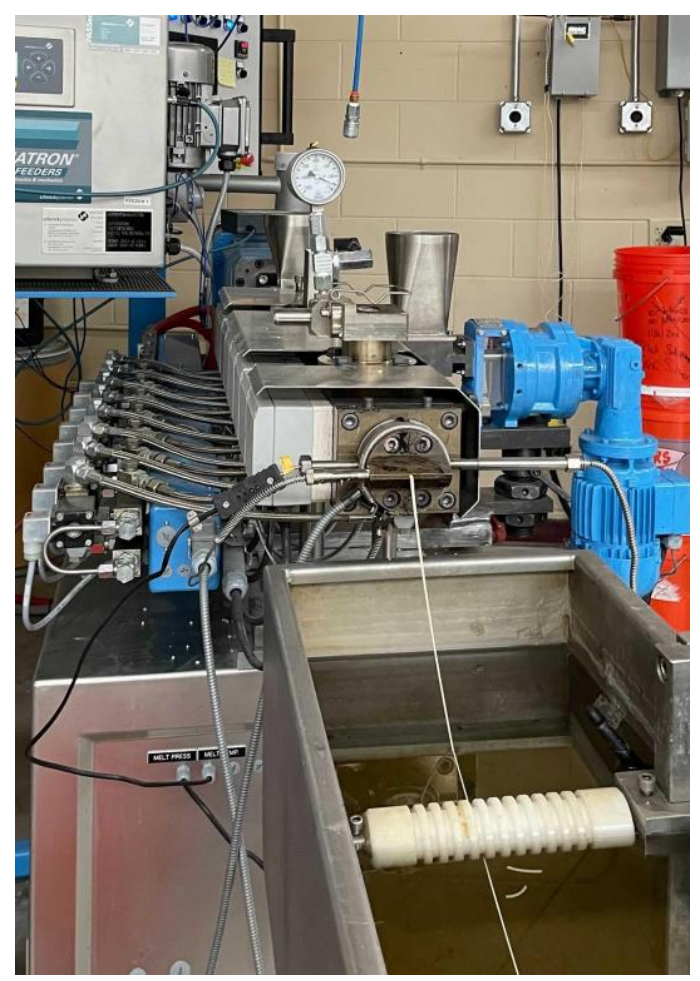

Figure 5: Extrusion of compounded material

\section{Single screw additive manufacturing}

A single-screw extruder 3D printer was employed to produce 3D PCs. This system allows the extrusion of materials that are difficult to supply in filament form [18]. This printer (Fused Form - FF600+) is able to extrude both pellets and filament from two separate nozzles. Pellets in the hopper can be transported to the throat of the printing head using vacuum, where the pellets are melted and extruded through the heated single screw. With the moving of the printing head, the molten materials are deposited on the print bed in a layer-by-layer manner [19]. In order to avoid 
interference caused by humidity, the pellets were dried at $60^{\circ} \mathrm{C}$ for 4 hours before poured into the hopper. A schematic of the printer can be seen in Fig. 6 where the heat shield and the outer shell of the barrel have been exposed to show the screw.

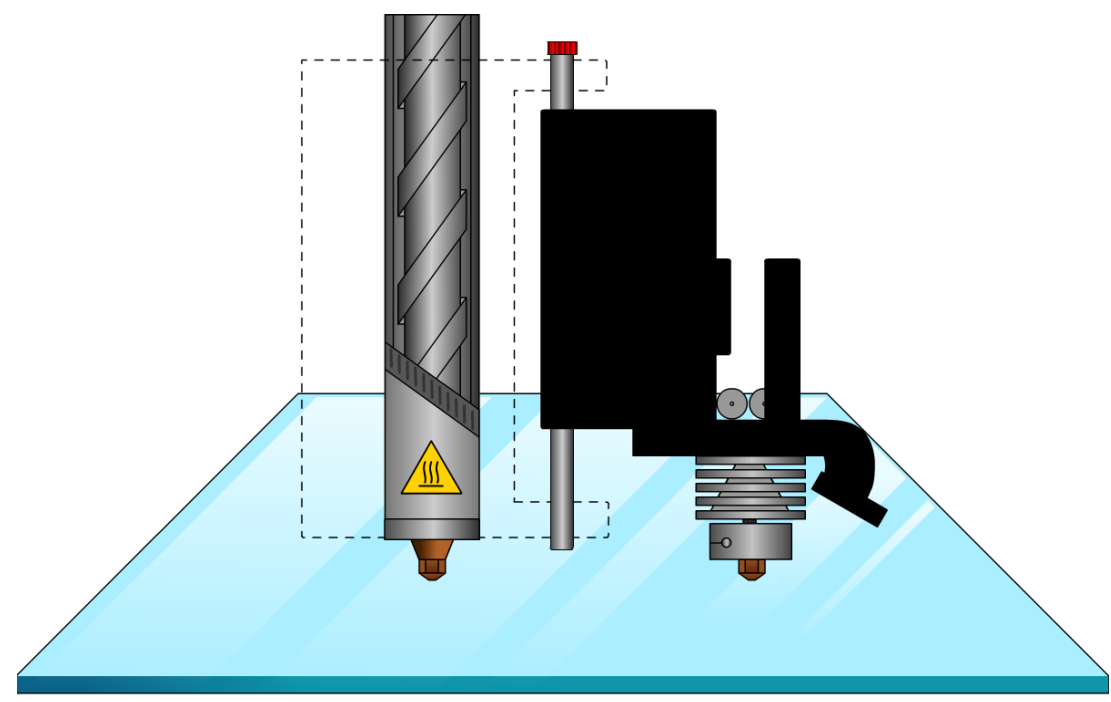

Figure 6: Schematic of single-screw extruder 3D printer

The general manufacturing process of the samples went as follows: the models generated through CAD software were converted into machine instructions using a slicing engine, and subsequently printed atop an aluminum sheet. This substrate was used to facilitate sample removal post printing, as well as enhancing signal detection in the wave propagation experimental setup. The toolpath files were created using the Simplify3D engine. The printing speed was selected by considering both printing efficiency and sample quality. It is worth noting that the matching of pellets melting speed and the printing speed plays an important role in the integrity of the printed objects and the wear of the screw. The relevant slicing parameters are shown in Table 2. Note that the print temperature chosen was $160^{\circ} \mathrm{C}$, as opposed to the $130^{\circ} \mathrm{C}$ used on the twin-screw process. Any temperature below this point would prove difficult to print, as the motor that drives the motion of the screw would seize. 
Table 2: List of relevant slicing parameters

\begin{tabular}{|c|c|}
\hline Parameter & Selected value \\
\hline Layer height [mm] & 0.25 \\
\hline Path width [mm] & 0.8 \\
\hline Extrusion multiplier & 1.8 \\
\hline $\begin{array}{c}\text { First layer height } \\
{[\%]}\end{array}$ & 100 \\
\hline $\begin{array}{l}\text { Print speed } \\
{[\mathrm{mm} / \mathrm{min}]}\end{array}$ & 500 \\
\hline Infill [\%] & 100 \\
\hline Infill orientation & {$\left[90^{\circ} 0^{\circ}\right]$} \\
\hline $\begin{array}{l}\text { Print temperature } \\
{\left[{ }^{\circ} \mathrm{C}\right]}\end{array}$ & 160 \\
\hline $\begin{array}{c}\text { Zone } 1 \text { temperature } \\
{\left[{ }^{\circ} \mathrm{C}\right]}\end{array}$ & 75 \\
\hline $\begin{array}{c}\text { Zone } 2 \text { temperature } \\
{\left[{ }^{\circ} \mathrm{C}\right]}\end{array}$ & 130 \\
\hline $\begin{array}{l}\text { Bed temperature } \\
{\left[{ }^{\circ} \mathrm{C}\right]}\end{array}$ & 60 \\
\hline $\begin{array}{c}\text { Outline perimeters } \\
{[-]}\end{array}$ & 3 \\
\hline
\end{tabular}

The first printed sample, as shown in Fig.7, was a rectangular slab for the dielectric constant characterization with the dimension of $100 \mathrm{~mm} \times 40 \mathrm{~mm} \times 9 \mathrm{~mm}$. This sample was tested as a Fabry-Parot (FP) cavity in a parallel plate waveguide. Two antennas fixed on the top plate were linked with a network analyzer, measuring transmission along the waveguide. The sample was placed in the center, surrounded by microwave absorption foam (as shown in Fig. 8). The complex dielectric constant was estimated by fitting the theoretical model to the experimental transmission spectrum. The model can be calculated from multi-beam interference theory [20]. Transmission $T=\left|\frac{4 n_{1} n_{2}}{\left(n_{1}+n_{2}\right)^{2}} \frac{e^{i k d}}{1-\left(\frac{n_{2}-n_{1}}{n_{1}+n_{2}}\right)^{2} e^{i 2 k d}}\right|^{2}$ can be calculated, where $n_{1}, n_{2}$ are the refractive indexes of air and the slab respectively, and $k$ represents the wave vector $k=n_{2} \frac{2 \pi f}{c}$, where $f$ is frequency, $c$ is the speed of light, and $d$ is the thickness of the sample. This differs from the expression in reference [20] by a "phase factor" $e^{i k d}$ in the numerator since there is absorption in our slab, represented by imaginary part of $n_{2}$. Taking the slab thickness into the theoretical 
transmission expression, $T$ can be fitted to the experiment transmission spectrum by adjusting the real and imaginary part of the dielectric constant of the slab. The fitting shows that the dielectric constant is $\epsilon_{2}=n_{2}^{2}=12.85+0.59 i$ from $10 \mathrm{GHz}$ to $12 \mathrm{GHz}$. The mismatch between experimental result and the theoretical curve, observed between $7 \mathrm{GHz}$ and $10 \mathrm{GHz}$, and after $12 \mathrm{GHz}$ is due to dispersion of the material. The real part of dielectric constant is the highest dielectric constant demonstrated to date for thermoplastic AM materials at microwave frequencies. The loss tangent is 0.046 , representing a low-loss dielectric.

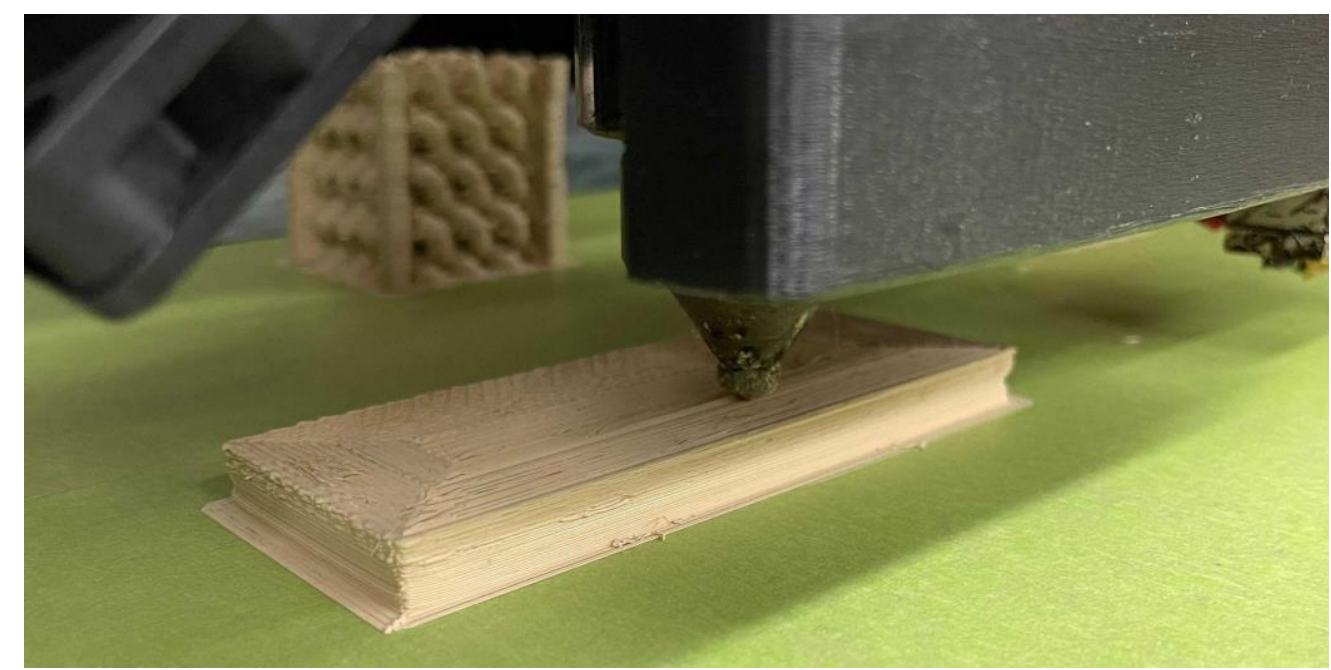

Figure 7: Rectangular slab produced to characterize dielectric constant of material

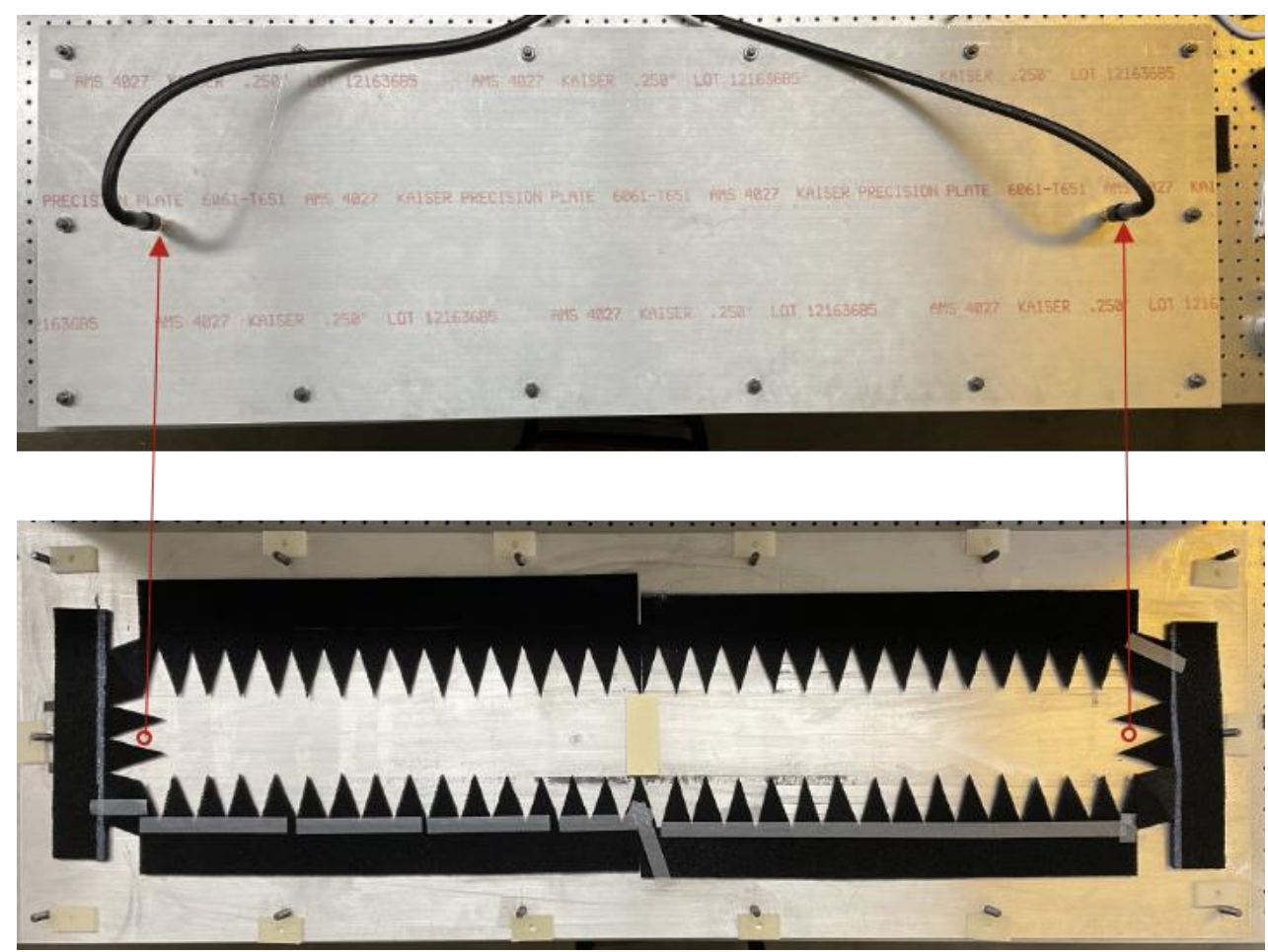

Figure 8: Testing setup for the first printed sample. Top: Parallel plate waveguide with two antennas linking network analyzer. Bottom: Parallel waveguide with top plate removed. Red circles are positions of two antennas. 


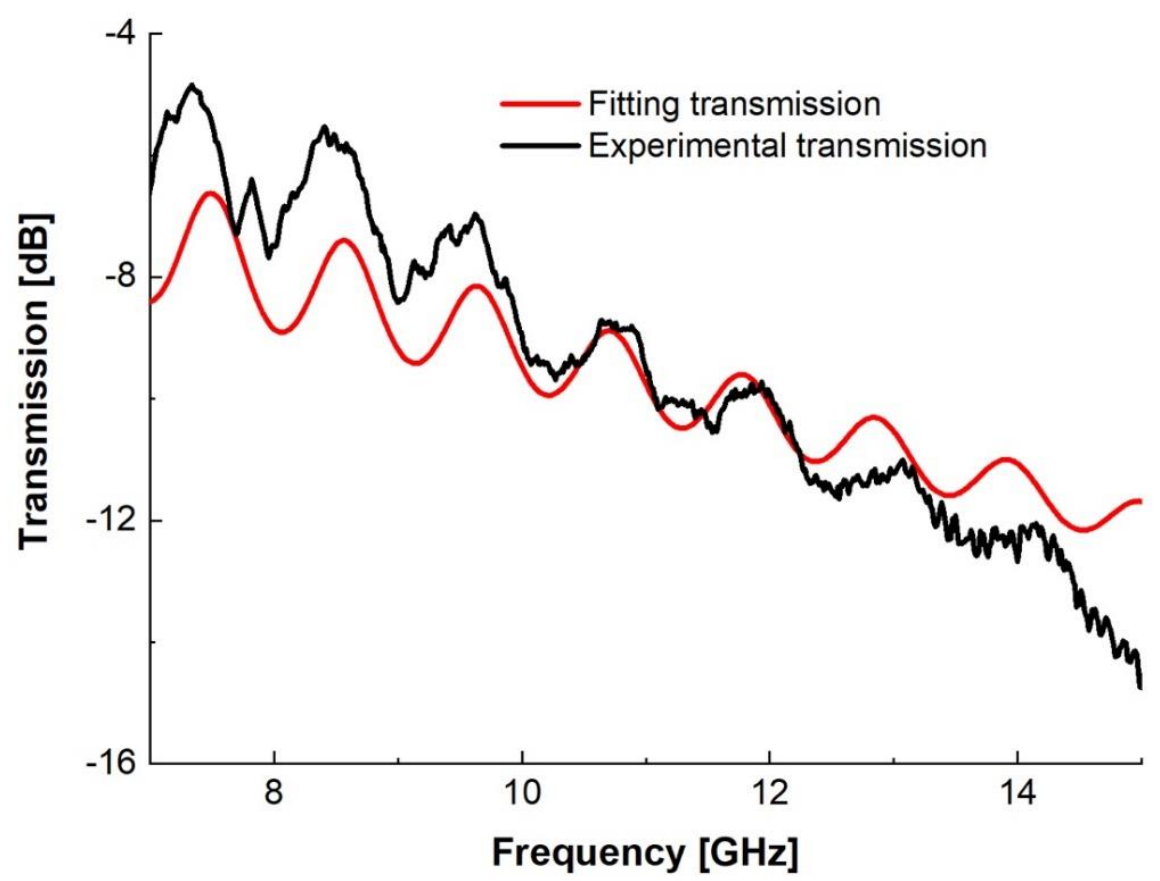

Figure 9: The experimental transmission and theoretical fitting transmission spectrum.

The second set of samples were prototypical 3D photonic crystals, inspired by the topological photonic crystal proposed by Yang, et al. [19]. This crystal has a simple cubic unit cell containing four rods connected in a tetrahedral geometry with the diameter of one rod reduced by $5 \%$ (Figure 1a), and the corresponding band structure is shown in Figure 10b.

a)

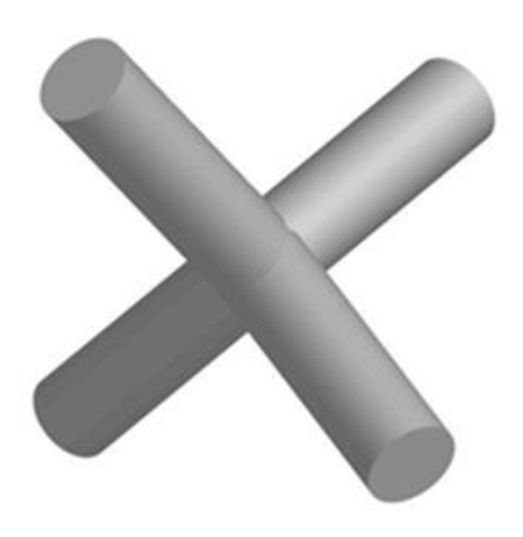

b)

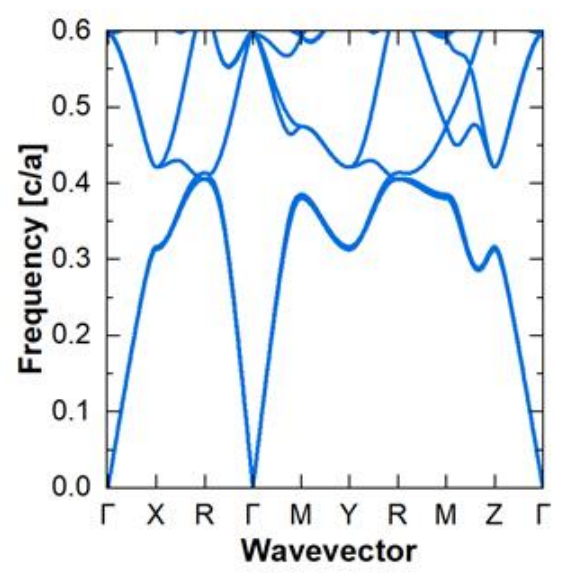

Figure 10: a) Unit cell of the 3D photonic crystal with unit cell length $\mathrm{a}_{0}$, and rod radius $\mathrm{r}=0.12 \mathrm{a}_{0}$.

b) Corresponding simulated band structure with the radius of one rod set to $0.95 \mathrm{r}$, and a dielectric constant of 12.9 [21] 
Initial attempts to reproduce the geometry resulted in poor fidelity to the original CAD file. The pillars in the structure were difficult to reproduce without the use of support structures in the four corners. The file was then modified to include built-in support, and this structure is displayed in Figure 11. The erratic strands that can be seen on the surface of the structure are the result of the inability to completely pause the dripping of materials in the screw printer due to residual pressure within the barrel during relocating, non-depositing movements of the gantry. These defects are unlikely to have an impact on the final performance of the part, as these are in the order of magnitude of fractions of a millimeter, while the wavelength in the frequency range of interest oscillates between 2 and $4.3 \mathrm{~cm}$.

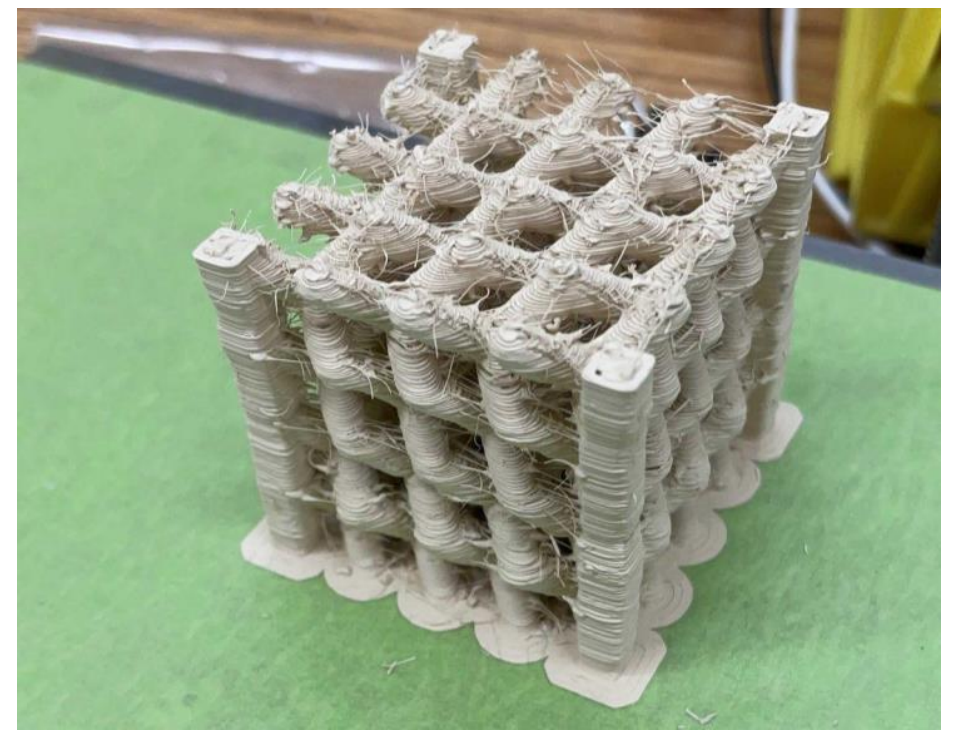

Figure 11: 3D Photonic Crystal

Additional attempts were made using the original CAD and support structures printed using PVA and PLA yielded undesirable results. Both PVA and PLA had poor adhesion to the surface of the composite, resulting in poor surface quality and failed support structure generation. An example can be seen in Figure 12. This image shows prints stopped prior to completion to illustrate the poor adhesion between support material and the composite. 


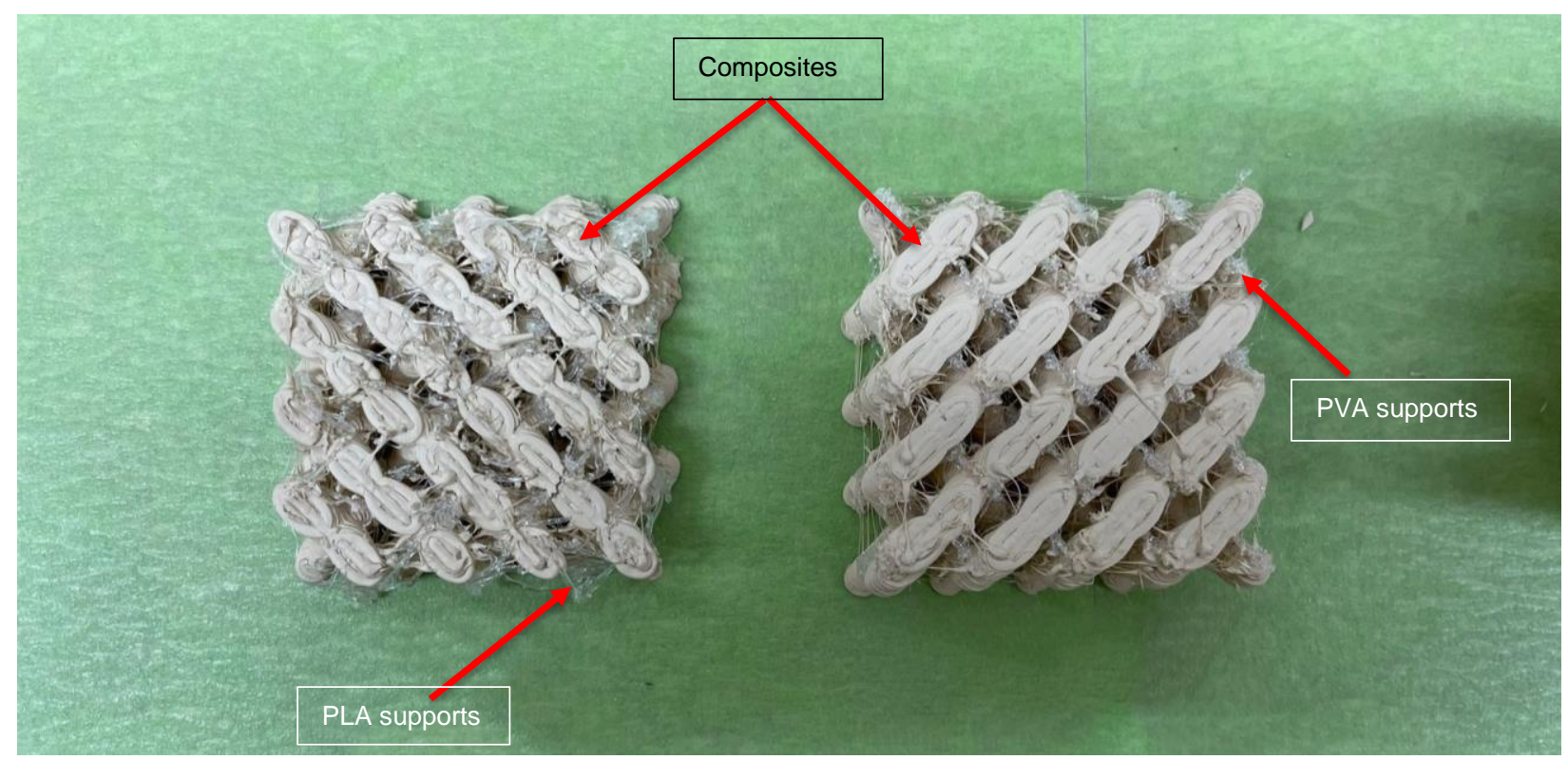

Figure 12: Partial print with PLA support structures (left) and PVA (right)

\section{Conclusions:}

The methodology of producing a small batch of the material using an internal batch mixer, and experimenting on it prior to scaling up production allowed the researchers to fine-tune the processing window while minimizing trial and error. The addition of plasticizer and surfactant required the material to be processed at $130^{\circ} \mathrm{C}$ in the twin screw process, producing homogeneous pellets of material. This temperature had to be adapted to $160^{\circ} \mathrm{C}$ for the single screw extrusion printing due to limitations of the machine. At this temperature, the viscosity of the material is comparable to the viscosity of neat ABS. By contrast, the material produced without plasticizer had a complex viscosity an entire order of magnitude larger.

Producing a slab of composite through 3D printing allowed measuring the complex dielectric constant of the material using a parallel plate microwave waveguide. The real part of the dielectric constant was measured to be 12.85 in the frequency range of $10 \mathrm{GHz}$ to $12 \mathrm{GHz}$, with a loss tangent of 0.046 , - the highest microwave dielectric constant reported to date for a thermoplastic AM composite. The complex geometry of the crystal requires the addition of support structures. The prints produced using support produced with PLA and PVA proved unsuccessful, given the poor adhesion between the composite and the support material. The alternative that yielded the highest fidelity to the original CAD file was the model that included the support pillars on the vertical edges of the structure and were produced using the composite itself. All structures had surface defects, resulting from the impossibility of stopping the flow of material during travel moves due to residual pressure within the barrel of the single screw extruder. These surface defects are likely to have little impact on the bulk properties of the photonic crystal, as their submillimeter dimensions are much smaller than the wavelength in the tested frequency range ( 2$4.3 \mathrm{~cm}$ ). Future work will involve the production of 3D photonic crystals with complex geometries unattainable with subtractive methods, and $3 \mathrm{D}$ characterization of the their photonic band structure. 
Acknowledgements:

This research was supported by the National Science Foundation through the University of Wisconsin Materials Research Science and Engineering Center (DMR-1720415). A.D. was supported by the National Science Foundation (REU grant CHE-1659223). The authors would also like to thank Dr. Thomas Mulholland for being the first point of contact for all parties involved during the very early stages of this project. 


\section{Bibliography}

[1] D. Isakov, C. J. Stevens, F. Castles, and P. S. Grant, "3D-Printed High Dielectric Contrast Gradient Index Flat Lens for a Directive Antenna with Reduced Dimensions," Advanced Materials Technologies, vol. 1, no. 6, Sep. 2016, doi: 10.1002/admt.201600072.

[2] H. Y. Jeong, E. Lee, S. C. An, Y. Lim, and Y. C. Jun, "3D and 4D printing for optics and metaphotonics," Nanophotonics, vol. 9, no. 5. De Gruyter, pp. 1139-1160, May 01, 2020. doi: 10.1515/nanoph-2019-0483.

[3] F. Castles et al., "Microwave dielectric characterisation of 3D-printed BaTiO3/ABS polymer composites," Scientific Reports, vol. 6, 2016, doi: 10.1038/srep22714.

[4] M. Yin, X. Yong Tian, H. Xue Han, and D. Chen Li, "Free-space carpet-cloak based on gradient index photonic crystals in metamaterial regime," Applied Physics Letters, vol. 100, no. 12, Mar. 2012, doi: 10.1063/1.3696040.

[5] J. Ren and J. Y. Yin, "3D-printed low-cost dielectric-resonator-based ultra-broadband microwave absorber using carbon-loaded Acrylonitrile Butadiene styrene polymer," Materials, vol. 10, no. 7, Jul. 2018, doi: 10.3390/ma11071249.

[6] D. v. Isakov, Q. Lei, F. Castles, C. J. Stevens, C. R. M. Grovenor, and P. S. Grant, "3D printed anisotropic dielectric composite with meta-material features," Materials and Design, vol. 93, pp. 423-430, Mar. 2016, doi: 10.1016/j.matdes.2015.12.176.

[7] M. S. Mattei et al., "Rapid Design, Fabrication, and Optimization of 3D Printed Photonic Topological Insulators."

[8] E. Goi, Z. Yue, B. P. Cumming, and M. Gu, "Observation of Type I Photonic Weyl Points in Optical Frequencies," Laser and Photonics Reviews, vol. 12, no. 2, Feb. 2018, doi: 10.1002/Ipor.201700271.

[9] L. Lu et al., "Experimental observation of Weyl points," Science, vol. 349, no. 6248, pp. 622-624, Aug. 2015, doi: 10.1126/science.aaa9273.

[10] S. Kirihara, Y. Miyamoto, and K. Kajiyama, "Fabrication of Ceramic-Polymer Photonic Crystals by Stereolithography and Their Microwave Properties."

[11] S. Kirihara, Y. Miyamoto, K. Takenaga, M. Wada Takeda, and K. Kajiyama, "Fabrication of electromagnetic crystals with a complete diamond structure by stereolithography." [Online]. Available: www.elsevier.com/locate/ssc

[12] Malas, Isakov, Couling, and Gibbons, "Fabrication of High Permittivity Resin Composite for Vat Photopolymerization 3D Printing: Morphology, Thermal, Dynamic Mechanical and Dielectric Properties," Materials, vol. 12, no. 23, p. 3818, Nov. 2019, doi: $10.3390 /$ ma12233818.

[13] Y. Wu, D. Isakov, and P. S. Grant, "Fabrication of composite filaments with high dielectric permittivity for fused deposition 3D printing," Materials, vol. 10, no. 10, Oct. 2017, doi: 10.3390/ma10101218.

[14] S. Uddin et al., "Effect of batio3 on the properties of pvc-based composite thick films," Materials, vol. 14, no. 18, Sep. 2021, doi: 10.3390/ma14185430.

[15] X. Quan, H. E. Bair, and G. E. Johnson, "Description of Polymers and Blends sample description"," 1989.

[16] "Handbook DSC. Netzsch GmbH \& Co," 2018. [Online]. Available: Handbook DSC. Netzsch GmbH \& Co 
[17] T. Hanemann, D. Syperek, and D. Nötzel, "3D printing of ABS barium ferrite composites," Materials, vol. 13, no. 6, Mar. 2020, doi: 10.3390/ma13061481.

[18] J. Gonzalez-Gutierrez, S. Cano, S. Schuschnigg, C. Kukla, J. Sapkota, and C. Holzer, "Additive manufacturing of metallic and ceramic components by the material extrusion of highly-filled polymers: A review and future perspectives," Materials, vol. 11, no. 5. MDPI AG, May 18, 2018. doi: 10.3390/ma11050840.

[19] Z. Yang, M. Xiao, F. Gao, L. Lu, Y. Chong, and B. Zhang, "Weyl points in a magnetic tetrahedral photonic crystal," Optics Express, vol. 25, no. 14, p. 15772, Jul. 2017, doi: 10.1364/oe.25.015772.

[20] M. Born, E. Wolf, "Principles of optics: electromagnetic theory of propagation, interference and diffraction of light," Physics Today, vol. 53, no. 10, 2000, doi: $10.1063 / 1.1325200$.

[21] S. G. Johnson, J. D. Joannopoulos, R. D. Meade, A. M. Rappe, K. D. Brommer, and O. L. Alerhand, "Photonic crystals: putting a new twist on light," 1997. [Online]. Available:

http://ab-initio.mit.edu/ 